\title{
Cálculo de la densidad de potencia eléctrica para el alumbrado público de las vías de la ciudad de Cuenca
}

\section{Electrical power density calculation for public street lighting in the city of Cuenca}

\author{
Pedro Andrés Villa Villa ${ }^{1}$, Franklin Mauricio Mayaguari Zhunio ${ }^{1}$ y Giovanni Santiago Pulla Galindo ${ }^{2}$ \\ Universidad de Cuenca $^{1}$ \\ Universidad de Católica de Cuenca ${ }^{2}$ \\ *pedro.villa2501@ucuenca.ec
}

DOI: https://doi.org/10.26871/killkana_tecnica.v3i1.370

\begin{abstract}
Resumen
La tendencia del mundo actual se orienta a la búsqueda de sistemas que consideren un uso racional y eficiente de la energía, en donde los recursos sean aprovechados al máximo, manteniendo la calidad de bienes y servicios. El sistema de alumbrado público no se encuentra exento de esta realidad, por lo que es necesario evaluarlo bajo un indicador de eficiencia energética que nos permita conocer si se dispone de un adecuado uso de la energía. El indicador que se empleó en este trabajo es el de la Densidad de Potencia para Alumbrado de vías (DPEA) propuesto por el Reglamento Técnico de Iluminación y Alumbrado Público (RETILAP), este indicador considera la carga total conectada para alumbrado y el área total iluminada en metros cuadrados $\left(\mathrm{W} / \mathrm{m}^{2}\right)$. El principal objetivo de este trabajo es presentar un resumen del cálculo de la DPEA para la red vial del área urbana de la ciudad de Cuenca y en base a ello identificar las vías que cumplen con tal criterio. Para lograr este objetivo, se realizó una medición de los parámetros necesarios para el cálculo de la DPEA y de esta forma presentar las vías que usan de forma eficiente la energía. Con la información obtenida, también se estableció una DPEA mínima que permite conocer si las vías están correctamente iluminadas. Sin embargo, dadas las diferentes condiciones bajo las cuales se realizaron las mediciones, los resultados deben ser analizados y contrastados en próximos trabajos de investigación.
\end{abstract}

Palabras clave: Eficiencia energética, alumbrado público, Densidad de Potencia para Alumbrado, Relación de Eficiencia Energética.

\begin{abstract}
The current world trend is oriented towards the search of rational and efficient use of energy systems, where the full advantage of resources are taken, preserving the quality of goods and services. The public lighting system is not exempted from this reality. It is necessary to evaluate it by using an energy efficiency indicator that allows knowing about the availability of adequate use of energy. The indicator used in this work is the Power Density for Street Lighting (DPEA), proposed by the Technical Regulation on Illumination and Street Lighting (RETILAP). This indicator considers the total power supplied for lighting and the total illuminated area in square meters $\left(W / \mathrm{m}^{2}\right)$. The main objective of this research is to present a summary on DPEA calculation for the urban area road system in the city of Cuenca, and on this basis, identify the roads meeting this criterion. In order to achieve this objective, a measurement of the parameters that are necessary to calculate the DPEA was carried out, and thus, it was possible to identify the roads using energy in an efficient manner. However, due to the different conditions under which the measurements were made, the results must be analyzed and contrasted in future investigations.
\end{abstract}

Key words: Energy efficiency, public lighting, Lighting Power Density, Energy Efficiency Ratio.

\section{INTRODUCCIÓN}

El sistema de alumbrado público constituye uno de los servicios fundamentales dentro de un país, debido a que brinda confort y seguridad a sus habitantes, permite el desarrollo de diferentes actividades en horarios nocturnos, activa la economía del lugar, y contribuye en la iluminación ornamental de ciertos espacios públicos.

Al ser el alumbrado público un servicio tan importante, requiere que sea evaluado bajo el criterio de la eficiencia energética [1], puesto que no siempre un mayor consumo energético equivale a un mejor servicio [2], motivo por el cual, en el presente trabajo se analiza la eficiencia energé- 
tica del sistema de alumbrado público del área urbana de la ciudad de Cuenca bajo el concepto de la Densidad de Potencia para Alumbrado de vías.

El criterio de la DPEA establece que aquellas vías cuyas velocidades de circulación sean inferiores a $60 \mathrm{Km} / \mathrm{h}$, es decir, aquellas diseñadas con el criterio de iluminancia no deben exceder los valores máximos de DPEA de la Tabla I. Estos valores han sido determinados con base en el valor de iluminancia promedio mantenida y el ancho de calzada correspondiente. Los valores no se deben exceder en el diseño ni posteriormente en la operación del sistema de alumbrado público [3].

TABLA I. Valores máximos de densidad de potencia eléctrica para alumbrado (DPEA) para vías vehiculares $\left(W / m^{2}\right)$ [3]

\begin{tabular}{|c|c|c|c|c|c|}
\hline \multirow{3}{*}{$\begin{array}{c}\text { Nivel } \\
\text { manteni- } \\
\text { do de } \\
\text { iluminan- } \\
\text { cia } \\
\text { promedio } \\
\text { lux (lx) }\end{array}$} & \multicolumn{5}{|c|}{$\begin{array}{l}\text { DENSIDAD DE POTENCIA }\left(W / m^{2}\right) \\
\text { según ancho de la calzada }(\mathrm{m})\end{array}$} \\
\hline & \multicolumn{5}{|c|}{ Ancho de calzada (m) } \\
\hline & $<6$ & 6 a 8 & 8.1 a 10 & 10.1 a 12 & 12.1 a 14 \\
\hline 3 & 0.29 & 0.26 & 0.23 & 0.19 & 0.17 \\
\hline 4 & 0.35 & 0.32 & 0.28 & 0.26 & 0.23 \\
\hline 5 & 0.37 & 0.35 & 0.33 & 0.3 & 0.28 \\
\hline 6 & 0.44 & 0.41 & 0.38 & 0.35 & 0.31 \\
\hline 7 & 0.53 & 0.49 & 0.45 & 0.42 & 0.37 \\
\hline 8 & 0.6 & 0.56 & 0.52 & 0.48 & 0.44 \\
\hline 9 & 0.69 & 0.64 & 0.59 & 0.54 & 0.5 \\
\hline 10 & 0.76 & 0.71 & 0.66 & 0.61 & 0.56 \\
\hline 11 & 0.84 & 0.79 & 0.74 & 0.67 & 0.62 \\
\hline 12 & 0.91 & 0.86 & 0.81 & 0.74 & 0.69 \\
\hline 13 & 1.01 & 0.94 & 0.87 & 0.8 & 0.75 \\
\hline 14 & 1.08 & 1.01 & 0.94 & 0.86 & 0.81 \\
\hline 15 & 1.12 & 1.06 & 1 & 0.93 & 0.87 \\
\hline 16 & 1.17 & 1.1 & 1.07 & 0.99 & 0.93 \\
\hline 17 & 1.23 & 1.17 & 1.12 & 1.03 & 0.97 \\
\hline 18 & 1.33 & 1.26 & 1.2 & 1.1 & 1.04 \\
\hline 19 & 1.4 & 1.33 & 1.26 & 1.17 & 1.1 \\
\hline 20 & 1.47 & 1.39 & 1.33 & 1.23 & 1.16 \\
\hline 21 & 1.55 & 1.46 & 1.39 & 1.29 & 1.22 \\
\hline 22 & 1.62 & 1.53 & 1.46 & 1.35 & 1.27 \\
\hline 23 & 1.69 & 1.6 & 1.53 & 1.41 & 1.33 \\
\hline 24 & 1.76 & 1.67 & 1.59 & 1.47 & 1.39 \\
\hline 25 & 1.83 & 1.73 & 1.66 & 1.53 & 1.45 \\
\hline 26 & 1.9 & 1.8 & 1.73 & 1.6 & 1.51 \\
\hline
\end{tabular}

Nota: Los valores de iluminancia para aplicar la tabla en el caso de diseños con base en criterio de luminancia de la calzada, serán los calculados por el diseñador, asociados al diseño.

El cálculo de la DPEA se realiza a partir de la carga total conectada para alumbrado y del área total por iluminar, esta relación se observa en la siguiente expresión:

$$
D P E A=\frac{\text { Cargatotal conectada para alumbrado }}{\text { Área total Iluminada }}\left[\frac{W}{m^{2}}\right][3]
$$

Las vías con velocidades superiores a los $60 \mathrm{Km} / \mathrm{h}$ y diseñadas con el criterio de luminancia presentarán un análisis diferente. La DPEA es sustituida por el concepto de Relación de Eficiencia Energética (EER - Energy Efficiency Ratio), cuyo cálculo se realiza en base a la potencia activa de la luminaria [3], la luminancia del área iluminada y el área iluminada. Esta relación se observa a continuación:

$$
E E R=\frac{P}{L \times S}\left[\frac{W}{\frac{c d}{m^{2}} m^{2}}\right][3]
$$

\section{MATERIAles y MÉTOdos}

La metodología empleada para evaluar la eficiencia energética del sistema de alumbrado público de la parte urbana de la ciudad de Cuenca se puede resumir en:

\section{A. Determinación del área y carga conectada para el alumbrado de vías}

En primera instancia se procede a calcular el área y la carga total conectada para el alumbrado de cada una de las vías de la ciudad, para ello se hace uso del programa ArcGIS, y de la base de datos proporcionada por el Departamento de Análisis y Sistemas Geográficos de Distribución (SIGADE) de la Empresa Regional CENTROSUR, que es la empresa prestadora del servicio de alumbrado público en la ciudad de Cuenca (Giovani Pulla Galindo, 2015).

La medición del área de las vías, así como la determinación de la carga conectada para el alumbrado de las mismas sigue un procedimiento sistemático, el mismo que se explica en el cálculo de estos parámetros y que requieren que se tomen en cuenta las siguientes consideraciones:

- No incluir las áreas destinadas a aceras o parterres en el cálculo de la DPEA.

- En el caso de los equipos para alumbrado (luminarias) que requieran el uso de balastros u otros dispositivos auxiliares para su operación, se considera un consumo del $10 \%$ de la potencia de la lámpara. [4]

\section{B. Cálculo de la DPEA}

En el inciso anterior se determino los parámetros necesarios para el cálculo de la DPEA, en donde la carga total conectada para el alumbrado de vías está expresada en vatios y el área total iluminada está definida en metros cuadrados, por lo que se procede a calcular la DPEA para la red vial de la ciudad de Cuenca. Las vías fueron clasificadas en: avenidas, calles y retornos de acuerdo con las características propias de cada una y a sus velocidades de circulación [5].

\section{Vías analizadas con el criterio de la EER}

La red vial de la ciudad de Cuenca cuenta con vías cuyas velocidades de circulación superan los $60 \mathrm{Km} / \mathrm{h}$, por lo que resulta necesario emplear el criterio de la EER para el análisis de la eficiencia energética. Las vías que son evaluadas bajo este criterio se observan en la Tabla II [5].

TABLA II. Vías de la ciudad de Cuenca analizadas bajo el criterio de la EER

\begin{tabular}{|l|c|}
\hline Nombre & $\begin{array}{c}\text { Velocidad } \\
(\mathrm{Km} / \mathrm{h})\end{array}$ \\
\hline Circunvalación Sur & 90 \\
\hline Enrique Arízaga Toral & 90 \\
\hline Panamericana Norte & 90 \\
\hline Panamericana Sur & 90 \\
\hline
\end{tabular}




\section{Análisis de la DPEA y EER}

Los valores de la DPEA correspondientes a las vías de la ciudad no deben superar una DPEA máxima para tener un uso eficiente de la energía, la DPEA máxima se calcula con ayuda de la Tabla III. Es necesario conocer el ancho de la vía analizada y el nivel mantenido de iluminancia promedio, el primero de estos parámetros se obtiene con ayuda del programa ArcGIS, teniendo en consideración que cuando se tengan anchos de vías diferentes a los mostrados en la Tabla I, se tomen los valores de ancho de calle de la columna inmediata a la que le antecede [6].

El nivel mantenido de iluminancia promedio presente en las vías varía de una a otra y depende exclusivamente de las características propias de cada una de ellas. En el RETILAP la asignación de una clase de iluminación (M1... M5) para una vía, se hace en función de las características de la vía, siendo las principales: la velocidad y el número de vehículos que circulan en una hora. Para la ciudad de Cuenca se establece las siguientes clases de iluminación:

TABLA III. Clases de iluminación de las vías de la ciudad de Cuenca

\begin{tabular}{|c|c|c|c|}
\hline $\begin{array}{c}\text { Clase de } \\
\text { iluminación }\end{array}$ & Avenidas & Calles & Retorno \\
\hline M1 & $90 \mathrm{~km} / \mathrm{h}$ & - & - \\
\hline M2 & $50-40 \mathrm{~km} / \mathrm{h}$ & $50 \mathrm{~km} / \mathrm{h}$ & - \\
\hline M3 & $30 \mathrm{~km} / \mathrm{h}$ & $40-30 \mathrm{~km} / \mathrm{h}$ & $30-20 \mathrm{~km} / \mathrm{h}$ \\
\hline M4 & - & $20-10 \mathrm{~km} / \mathrm{h}$ & $10 \mathrm{~km} / \mathrm{h}$ \\
\hline
\end{tabular}

En función de las clases de iluminación se establecen los valores de iluminación promedio, estos valores se consiguen luego de multiplicar los valores de iluminancia por el coeficiente $\mathrm{R}$ del pavimento utilizado, con un valor de 15 cuando este no se conozca [7]. Tabla IV.

TABLA IV. Valores de luminancia e iluminancia por clase de iluminación para tráfico motorizado

\begin{tabular}{|c|c|c|}
\hline $\begin{array}{c}\text { Clase de } \\
\text { Iluminación }\end{array}$ & $\begin{array}{c}\text { Luminancia } \\
\text { promedio } \\
\left(\frac{c d}{m^{2}}\right)\end{array}$ & $\begin{array}{c}\text { Iluminancia } \\
\text { promedio } \\
\left(\frac{l m}{m^{2}}\right)\end{array}$ \\
\hline M1 & 2.0 & 30 \\
\hline M2 & 1.5 & 23 \\
\hline M3 & 1.2 & 18 \\
\hline M4 & 0.8 & 12 \\
\hline M5 & 0.6 & 9 \\
\hline
\end{tabular}

Con la información expuesta anteriormente se procede a determinar el valor de la iluminancia promedio para las vías de la ciudad y con ello el valor de la DPEA máxima. El valor máximo de EER aceptado será $0,8 \mathrm{~W} / \mathrm{cd}$. Los valores para cálculo serán tomados de la información suministrada por el fabricante [3].

\section{E. DPEA mínima}

En el RETILAP se establecen los valores máximos de DPEA para una vía, sin embargo, estos valores no permiten conocer si una vía se encuentra iluminada correctamente por lo que en este apartado se propone calcular una DPEA mínina para cada vía de la ciudad. De esta forma si los valores de la DPEA obtenidos en los incisos anteriores se encuentran dentro del siguiente rango: $D P E A_{m i n}-$ $D P E A_{\max }$ se podría concluir que la vía se encuentra iluminada correctamente y posee un uso racional y eficiente de la energía [8].

Para el cálculo de la $D P E A_{m i n}$ es necesario conocer el valor de la carga total mínima conectada para alumbrado de una vía, y el área total iluminada, este último valor ya se conoce de las mediciones realizadas en los párrafos anteriores, mientras que la carga mínima se determina con la siguiente expresión:

En donde:

$$
P_{\text {min }}=E \times A / \eta[9]
$$

$P_{\text {min }}=$ Potencia mínima de una vía $(\mathrm{W})$

$\mathbf{E}$ = Iluminancia (lux)

A = Área total iluminada $\left(\mathrm{m}^{2}\right)$

$\eta=$ Eficacia luminosa $(\mathrm{lm} / \mathrm{W})$.

La ecuación dice que, conociéndose los luxes que requiere una vía, el área de la misma y la eficacia de las luminarias se puede conocer la potencia necesaria. Sabiendo esto se procede a calcular la potencia mínima para iluminar cada una de las vías de la cuidad y con ello la $D P E A_{\min }$ mediante la siguiente expresión:

$$
D P E A_{\text {min }}=\frac{\text { Cargatotal mnima conectada para alumbrado }}{\text { Área total Iluminada }}\left(\frac{W}{\mathrm{~m}^{2}}\right)
$$

\section{RESUlTADOS}

A. Clase de iluminación de las vías de la ciudad de Cuenca

La ciudad de Cuenca cuenta con 2461 vías repartidas en avenidas, calles y retornos [5]. En la Tabla V, se muestra el número de vías que pertenecen a cada clase de iluminación.

TABLA V. Clase de iluminación de las vías de la ciudad de Cuenca

\begin{tabular}{|c|c|c|c|c|}
\hline $\begin{array}{c}\text { Clase de } \\
\text { iluminación }\end{array}$ & Avenidas & Calles & Retornos & Total \\
\hline M1 & 4 & 0 & 0 & 4 \\
\hline M2 & 31 & 19 & 0 & 50 \\
\hline M3 & 24 & 399 & 58 & 481 \\
\hline M4 & 0 & 1402 & 524 & 1926 \\
\hline
\end{tabular}

\section{B. Análisis de la norma de eficiencia energética}

Las vías analizadas bajo el criterio de la DPEA tienen una clase de iluminación M2, M3 y M4. En la Tabla VI, se muestra el número total de avenidas, calles y retornos de la cuidad que estarían cumpliendo con los valores estipulados en la norma, notándose que la gran parte de las vías poseen un uso eficiente de la energía. Cabe indicar que el porcentaje de las vías que cumplen con la norma puede incrementarse si se considera la potencia menor de las lámparas de doble nivel de potencia. 
TABLA VI. Resultados del análisis de la DPEA

\begin{tabular}{|l|c|c|c|}
\hline & Total & $\begin{array}{c}\text { Uso } \\
\text { eficiente } \\
\text { de la } \\
\text { energía }\end{array}$ & $\begin{array}{c}\text { Uso inefi- } \\
\text { ciente de } \\
\text { la energía }\end{array}$ \\
\hline Avenidas & 55 & $91 \%$ & $9 \%$ \\
\hline Calles & 1820 & $68 \%$ & $32 \%$ \\
\hline Retornos & 582 & $55 \%$ & $45 \%$ \\
\hline
\end{tabular}

Existen vías que requieren una mayor iluminación debido a la presencia de locales comerciales, lugares inseguros, parques, plazas y zonas de afluencia turística; dando lugar a una DPEA superior a la establecida en la Norma y a una sobre iluminación. El cálculo de una DPEA mínima permite determinar cuáles vías están correctamente iluminadas. En la Tabla VII se observa el porcentaje de vías con una adecuada iluminación.

TABLA VII. Análisis de la iluminación de las vías

\begin{tabular}{|c|c|c|c|}
\hline $\begin{array}{c}\text { Tipo de } \\
\text { calle }\end{array}$ & $\begin{array}{c}\text { Bien Ilu- } \\
\text { minadas }\end{array}$ & $\begin{array}{c}\text { Sobre ilu- } \\
\text { minadas }\end{array}$ & $\begin{array}{c}\text { Mal Ilu- } \\
\text { minadas }\end{array}$ \\
\hline Avenidas & 91 & 9 & 0 \\
\hline Calles & 66 & 32 & 2 \\
\hline Retornos & 54 & 45 & 1 \\
\hline
\end{tabular}

La mayoría de vías estarían bien iluminadas, las vías sobre iluminadas representan un considerable porcentaje debido a que algunas de ellas presentan requerimientos especiales como los que se mencionaron con anterioridad, en cuanto al porcentaje de vías mal iluminadas es mínimo.

Las vías cuyas velocidades de circulación superan los 60 $\mathrm{Km} / \mathrm{h}$ son analizadas bajo el criterio de la EER. Estas vías tienen una clase de iluminación M1 y son las siguientes: Circunvalación Sur, Enrique Arizaga Toral, Panamericana Norte y Panamericana Sur.

Conocidos los valores de EER, se procede a verificar si se estaría cumpliendo con el valor máximo establecido en el RETILAP que es $0.8 \mathrm{~W} / \mathrm{cd}$. En la Tabla 8 se aprecia los resultados de este análisis, evidenciándose que todas las vías estarían cumpliendo con el criterio de eficiencia energética [8].

TABLA VIII. Análisis de la EER

\begin{tabular}{|l|l|l|}
\hline Nombre & $\begin{array}{l}\text { EER } \\
(W / c d)\end{array}$ & $\begin{array}{l}\text { Eficiencia } \\
\text { energéti- } \\
\text { ca }\end{array}$ \\
\hline Circunvalación Sur & 0.28 & SI \\
\hline Enrique Arizaga Toral & 0.48 & SI \\
\hline Panamericana Norte & 0.44 SI & \\
\hline Panamericana Sur & 0.72 & SI \\
\hline
\end{tabular}

\section{CONCLUSIONES}

Una DPEA que cumplen con los valores estipulados en la norma no garantiza un nivel adecuado de iluminancia debido a que se puede tener una apropiada DPEA y un bajo nivel de iluminación o una adecuada DPEA y una correcta iluminación, por lo que es necesario establecer una densidad de potencia mínima para saber en qué escenario se encuentra tal como se propuso en este trabajo.
Una densidad de potencia superior a la máxima establecida en la norma ocasiona que la vía en estudio esté sobre iluminada y tenga un probable mal uso de la energía. Sin embargo, existen vías con requerimientos especiales que demandan la instalación de luminarias con potencias superiores a las requeridas para sus características.

Las avenidas analizadas con el criterio de DPEA, el $9 \%$ de ellas estarían superando los valores máximos de densidad de potencia. Por otro lado, el $91 \%$ de las avenidas estarían con un adecuado uso de la energía y disponen de una correcta iluminación, lo cual es de gran importancia ya que se tratan de vías con una importante presencia de vehículos y peatones.

El $68 \%$ del total de las calles tendrían valores de DPEA permitidos por la norma, existiendo un $66 \%$ de calles bien iluminadas y $2 \%$ mal iluminadas, el $32 \%$ estarían superando los valores de la DPEA de la norma.

Los retornos que no estarían cumpliendo con el criterio de eficiencia energética representan un $45 \%$ del total de los retornos. Del $55 \%$ de retornos que estarían cumpliendo con el criterio de la DPEA tan solo el $1 \%$ no cumplirían con los parámetros mínimos de iluminación establecidos en la regulación 005/2014 del ARCONEL.

Una DPEA superior a la establecida en la norma de algunas vías de la ciudad se debe a requerimientos especiales tales como: seguridad, ornamentación, desarrollo de actividades nocturnas y presencia de locales comerciales.

\section{AGRADECIMIENTOS}

Este trabajo es el resultado de la Tesis de grado, para la obtención del título de Ingeniero Eléctrico de los dos primeros autores que se realizó gracias al convenio existente entre la Universidad de Cuenca y la Empresa Eléctrica Regional Centro Sur C.A., por lo que se agradece a estas instituciones, a los funcionarios y al Departamento de Alumbrado Público de la CENTROSUR por al apoyo brindado.

\section{REFERENCIAS}

[1] G. S. Pulla-Galindo, "Evaluación energética del alumbrado público en la ciudad de Cuenca," 2013.

[2] I. P. Genovez-Zúñi, "Eficiencia energética en el servicio de alumbrado público del Ecuador," 2015.

[3] Empresa de Energia de Boyaca SA, "Reglamento Tecnico de Iluminación y Alumbrado Publico 'RETILAP'," in RETILAP, 2010.

[4] ARCONEL, "Regulación no. 005/14 "prestación del servicio de alumbrado público"," tech. rep., 2014.

[5] Ilustre Municipalidad de Cuenca, "Plan de Desarrollo y Ordenamiento Territorial del Cantón Cuenca," tech. rep., Cuenca, 2015.

[6] Norma Oficial Mexicana, Eficiencia energética para sistemas de alumbrado en vialidades. 2013.

[7] Ministerio de Industria, Energia y Turismo, "Guía Técnica de aplicación: Eficiencia Energética en Instalaciones de Alumbrado Exterior," tech. rep., Madrid, 2010. 
[8] P. Villa and F. Mayaguari, Cálculo de la densidad de potencia de eléctrica para vías de alumbrado público de la ciudad de Cuenca. 2017.

[9] Rapidtables, "Rapidtables-Watts to lux (lx) ."

Recibido: 3 de diciembre de 2018

Aceptado: 10 de marzo de 2019 
